\title{
Analysis of Game Influence Factors of Martial Art Event Competition
}

\author{
Kaihua $\mathrm{Xu}^{1}$, Yali $\mathrm{Xu}^{2}$ \\ ${ }^{1}$ Police Training Management Department, Jiangxi People's Police College, Nanchang, 330103, \\ China \\ ${ }^{2}$ School of Physical Education, Jiangxi University Of Traditional Chinese Medicine, Nanchang, \\ 330004, China
}

Key words: Competitive martial art, Winning element, Wining rule, Game theory.

\begin{abstract}
This paper analyzes game theory of winning rules of competitive martial art event from the macroscopic and microcosmic perspective and looks for athletes; selection of confrontation/non-confrontation strategy so as to strongly promote improvement of competitive martial art training level. This theory is improved to scientific theory level, which contributes to guiding martial art competition and exempting unnecessary tortuous path. Besides, training has strong scientificity. Meanwhile, martial art events can develop continuously. Thus, this paper provides theoretical support and has certain practical guidance significance.
\end{abstract}

\section{Introduction}

Martial art competition belongs to a popular sport activity, and the major objective is to create good competition results. Gaining excellent performance is the starting point and also the attaching point of competition activity in current stage. Game theory contains a series of winning rules which focus on the objective of gaining excellent performance and follow competitive sport development direction. Meanwhile, the basic conditions of winning must be met. In essence, winning rules are the fundamentals and also the most important principles which must be followed in economic practice. Winning rules are an important constituent part of game theory. Each stage of competition practice process must meet fundamental needs of winning rules, such as material selection and training stage as well as management and restriction stages. Competitive martial art has been in the mature stage in training aspect, and the competition ability also reaches mature period. Organization and management method of competition also approach to perfect stage. Thus, it is crucial to study winning rules of competitive martial art events.

\section{Confrontation form of competitive martial art under game conditions}

Competitive sport is wrestling confrontation event in skill-based exercise scope. The competition rules are differentiated according to weight, and the confrontation mode is one-to-one mode. The competition has the nature of absolute victory. Or score is used to judge the win or lose. The confrontation parties are reflected in five elements: exercise intelligence, psychological factor, tactics, technological factor and physical ability. The five elements embody the entire ability of an athlete and can better embody competition ability of an athlete, i.e. the ability to defeat the opposite side.

The competition result of competitive martial art event is generally decided by three aspects: behavior score of competitive martial art athlete, motion behavior score of the opponent, competition result judgment method. Most of all, competitive ability of an athlete in the competition process is the intrinsic factor of his competition result. This is the root cause. However, the performance of an athlete is influenced by the opponent's competitive ability. This is event the fundamental restriction, as shown in Fig.1. 


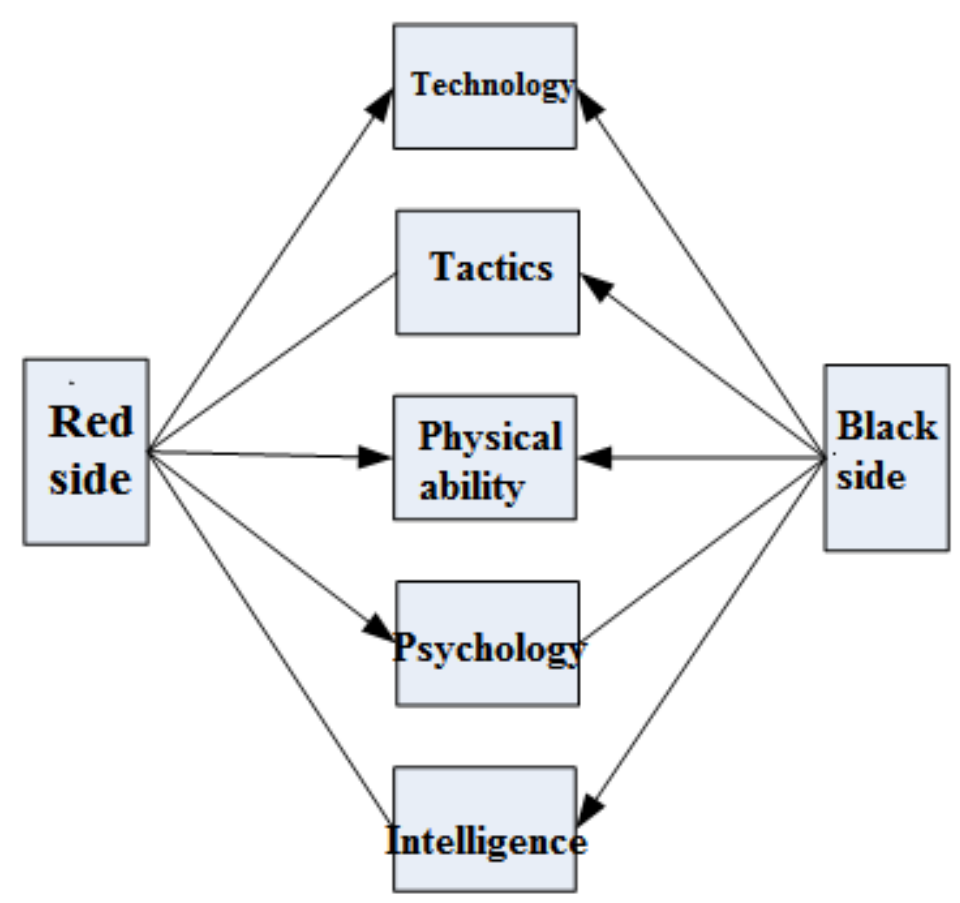

Fig.1 Confrontation manifestation pattern of competitive martial art athlete

\section{Game analysis of competitive martial art}

In the whole competition process of competitive martial art, the five elements are not isolated, but mutually influenced and promoted. They also have hierarchy on the whole. The important basic part is athlete's physical quality, form and somatic function. The basic composition of athlete's tactics is competitive technology. Tactics provides strong guarantee for giving full play to technology. Athletics intelligence belongs to decision making system and flexible coping system of athlete's tactics and technology.

\section{Hypothesis}

1) Set of Game participants. Here, we suppose there are only two athletes in a competition. We define the set $\mathrm{I}=\{\mathrm{I} 1, \mathrm{I} 2\}$, where I1 represents Athlete 1 , and I2 represents Athlete 2 . The ability of both is absolute, and their ability is close. We also suppose Athlete 1 and Athlete 2 are rational and selfish in the competition process. The information expressed by I1 and I2 has the same basic value r, same monopoly value $\mathrm{m}$, same synergy value $\mathrm{s}$ and partly negative effect $\mathrm{h}$ brought by information supplier.

2) Analysis of strategy set: the strategies in competition process are usually classified into two types: non-confrontation and confrontation. Thus, we define a strategy set $\mathrm{Sij}=\{$ non-confrontation $\mathrm{H}$, confrontation $\mathrm{J}$ \}, $\mathrm{i}=1$, 2. 1 represents Athlete 1 and 2 represents Athlete 2. $\mathrm{j}=1,2$ means the strategy adopted is non-confrontation 1 and non-confrontation 2. In other words, S11= non-confrontation, which represents non-confrontation strategy of Athlete 1 . S12=confrontation, which represents confrontation strategy of Athlete 1. S21= non-confrontation, which represents non-confrontation strategy of Athlete 2. S22= confrontation, which represents confrontation strategy of Athlete 2 .

3) Revenue function analysis. Generally speaking, revenue function $U$ is the effect after game party executes his strategy. The common formula is $\mathrm{U}=(\mathrm{p}-\mathrm{c}) \mathrm{Q}^{+} \varphi$. $\mathrm{Q}$ is total output; $\mathrm{P}$ is price. The fixed cost of all athletes is deemed as sunk cost which is not included. Both athletes have same unit cost c. $\varphi$ ( r,m,s,h ) refers to utility function of athletes' application of non-confrontation strategy. $r$ refers to the revenue brought by gaining technology element. $\mathrm{m}$ refers to high revenue brought by physical ability. s refers to non-conformation revenue brought by athletes' non-confrontation. $\mathrm{h}$ refers to 
information risk. The strategy profile of each athlete is stipulated, i.e. vector factor which consists of strategies of all athletes.

\section{Revenue function relationship of various strategy profiles}

Total game probability in the playground is $\mathrm{Q}=\mathrm{Q} 1+\mathrm{Q} 2$. According to the specific condition of playground, we set the probability function as follows:

$$
\mathrm{P}(\mathrm{Q})=\mathrm{a}-\mathrm{bQ}=\mathrm{a}-\mathrm{b}(\mathrm{Q} 1+\mathrm{Q} 2)
$$

1) Under non-intervention assumption condition, two athletes confront each other freely (i.e. both adopt confrontation strategy). Due to free confrontation in the process, the nature of athletes decides both sides regard result maximization as their final purpose to implement the service or production behavior. Under such assumption condition, the result functions of both athletes are as follows:

$$
\begin{gathered}
\mathrm{U} 1=\mathrm{PQ} 1-\mathrm{cQ} 1+\varphi_{1} \varphi_{1=\mathrm{r}+\mathrm{m}\}} \\
\mathrm{U} 2=\mathrm{PQ} 2-\mathrm{cQ} 2+\varphi_{1}
\end{gathered}
$$

Formula (1) is substituted into two result functions (2) and (3), respectively. Then, the derivative of $\mathrm{Q}$ of either athlete is solved. The only maximum value point (i.e. respective result maximization point of both parties) of the function can be figured out. In such case, the equilibrium quantity is Q1= $\mathrm{Q} 2=\frac{a-c}{3 b}$. The maximized result is $\mathrm{U} 1=\mathrm{U} 2=\frac{(a-c)^{2}}{9 b}+\mathrm{r}+\mathrm{m}$.

2) Both athletes adopt non-confrontation strategy. If both sides do not carry out confrontation strategy, both athletes carry out non-confrontation mode in order to create whole result maximization. In such case, the result function is

$$
\mathrm{U}=\mathrm{pQ}-\mathrm{cQ}=-\mathrm{bQ} 2+(\mathrm{a}-\mathrm{c})+\varphi_{2} \quad\left(\varphi_{2}=2 \mathrm{r}+\mathrm{s}-\mathrm{h}\right)
$$

The optimal result is $\operatorname{Umax}=\frac{(a-c)^{2}}{4 b}+2 \mathrm{r}+\mathrm{s}-\mathrm{h}$.

3) Unilateral destruction of confrontation equilibrium. Although both athletes gain larger result when they adopt non-confrontation strategy, both sides are rational and selfish in the confrontation and want to achieve their result maximization. Such non-confrontation equilibrium state is in the turmoil state, because the production of both sides is within the scope of game model. When one side destroys equilibrium, lager result will be obtained. In other words, If Athlete 1 first destroys the equilibrium state, Athlete 2 is still in the non-confrontation state. The result of Athlete 2 will change, i.e. Q1= $\frac{a-c}{4 b}$, Q2 $=\frac{a-c}{3 b}$. The output and result of Athlete 2 are Q2 $=\frac{3(a-c)}{8 b}, \mathrm{U} 2=\frac{(a-c)^{2}}{64 b}+2 \mathrm{r}+\mathrm{m}$. The output and result of Athlete 1 are $\mathrm{Q} 1=\frac{a-c}{4 b}, \mathrm{U} 1=\frac{(a-c)^{2}}{32 b}+\mathrm{r}-\mathrm{h}$.

\section{Game matrix}

The general influence of five elements in competitive martial art event is sorted according to impotence as follows: technology, physical ability, psychology, tactics and intelligence. The details are shown in Table 1.

Table 1. Importance of five elements in competitive martial art event

\begin{tabular}{|c|c|c|c|c|c|}
\hline Five elements & Physical ability & $\begin{array}{c}\text { Technolog } \\
\mathrm{y}\end{array}$ & Tactics & $\begin{array}{c}\text { Psycholog } \\
\mathrm{y}\end{array}$ & $\begin{array}{c}\text { Intelligenc } \\
\mathrm{e}\end{array}$ \\
\hline Frequency & 156 & 138 & 137 & 113 & 71 \\
\hline Weight coefficient & 0.26 & 0.22 & 0.22 & 0.18 & 0.12 \\
\hline
\end{tabular}


Technology is the most important element in competitive martial art and in the core position among the five elements, followed by good psychology, physical ability, intelligence and tactics.

A game matrix can be gained through revenue function calculation and importance degree of competitive martial art elements (see Table 2).

Table 2. Athlete's non-confrontation game matrix charm

\begin{tabular}{|c|c|c|c|}
\hline \multirow{2}{*}{ Participant } & \multicolumn{3}{|c|}{ Athlete 2} \\
\cline { 2 - 4 } \multicolumn{1}{|c|}{ Revenue } & \multicolumn{2}{|c|}{ Non-confrontation } & Confrontation \\
\hline \multirow{3}{*}{ Athlete 1} & $\begin{array}{c}\text { Non-confrontatio } \\
\mathrm{n}\end{array}$ & $\frac{(a-c)^{2}}{8 b}+2 \mathrm{r}+\mathrm{s}-\mathrm{h}, \frac{(a-c)^{2}}{8 b}+2 \mathrm{r}+\mathrm{s}-\mathrm{h}$ & $\frac{3(a-c)^{2}}{32 b}+\mathrm{r}-\mathrm{h}, \frac{9(a-c)^{2}}{64 b}+2 \mathrm{r}+\mathrm{m}$ \\
\cline { 2 - 5 } & Confrontation & $\frac{9(a-c)^{2}}{64 b}+2 \mathrm{r}+\mathrm{m}, \frac{3(a-c)^{2}}{32 b}+\mathrm{r}-\mathrm{h}$ & $\frac{(a-c)^{2}}{9 b}+\mathrm{r}+\mathrm{m}, \frac{(a-c)^{2}}{9 b}+\mathrm{r}+\mathrm{m}$ \\
\hline
\end{tabular}

\section{Influence rules of competitive martial art game}

1) When $\frac{(a-c)^{2}}{9 b}+r+m \geq \frac{(a-c)^{2}}{8 b}+2 r+s-h$, monopoly value of information reaches extreme high state, while the synergy value of information is relatively low. Both athletes consider they can create better value harvest if they implement conformation strategy. In this way, there is only pure strategy Nash equilibrium as follows: \{confrontation J, confrontation J\}, i.e. $\left\{\frac{(a-c)^{2}}{9 b}+\mathrm{r}+\mathrm{m}, \frac{(a-c)^{2}}{9 b}+\right.$ $\mathrm{r}+\mathrm{m}\}$. Seeing from the above model, Athlete 1 may gain the first-hand information about market information change through his informatization management and apply his ability to handle operation problem. Meanwhile, he considers if he adopts non-confrontation strategy, information resource provided by Athlete 2 may be not helpful to him. At this moment, information resource mastered by Athlete 1 has high monopoly value. Thus, Athlete 1 will choose confrontation strategy. Since Athlete 1 adopts confrontation strategy, Athlete 2 will not actively offer his information resource to Athlete 1 . In such case, both athletes will certainly carry out confrontation strategy, because the nature of both sides is selfish.

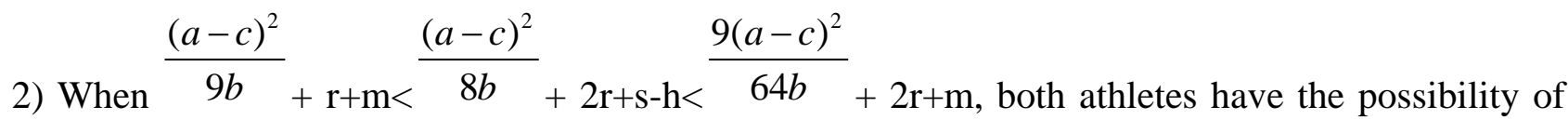
non-confrontation. However, non-confrontation value cannot eliminate negative effect generated by non-confrontation information provider. In this way, sole pure strategy Nash equilibrium appears \{no sharing, no sharing\}. The root cause is that when both athletes achieve sharing, external incentive factor must be gained, or the reputation of both sides or long-term non-confrontation trust accumulation serves as the condition to achieve non-confrontation strategy. To be specific, before Athlete 1 shares his information resource with Athlete 2, he may think if Athlete 2 transmits internal information resource to the opponent, the loss caused is larger than the value generated by Athlete 2's information resource. In addition, Athlete 1 will worry if he does not adopt non-confrontation strategy and Athlete 2 does not confront, he will suffer large loss. Thus, for the sake of safety, Athlete 1 will choose competitive strategy. When Athlete 2 sees Athlete 1 implements competition method, Athlete 2 will change original non-confrontation mode to competitive strategy. Even cutthroat competition will occur for the purpose of revenge.

3) When $\frac{(a-c)^{2}}{8 b}+2 \mathrm{r}+\mathrm{s}-\mathrm{h}>\frac{9(a-c)^{2}}{64 b}+2 \mathrm{r}+\mathrm{m}$, synergy value brought by non-confrontation is much greater than the monopoly value. Both athletes will adopt non-confrontation strategy to generate win-win effect. At this moment, Nash equilibrium is \{non-confrontation $\mathrm{H}$, non-confrontation $\mathrm{H}$ \}. 
Such form is the best method for both athletes to achieve giving maximization. Without external insensitive, both athletes adopt non-confrontation strategy beneficial to them. The information resource they share can generate the maximum coordination value. In other words, information resources of both sides are synthesized to create larger value. Here, a technical information resource is included. For example, both athletes will encounter the same technical problem. However, the problems cannot be well solved only through the strength of one side. If the problem handling method is used as resource sharing non-confrontation, both sides may handle such technical problems together. In this way, both sides create the maximum benefit and can implement non-confrontation method.

\section{Conclusions}

This paper studies features of competitive martial art and athletes' macroscopic and microcosmic athletics environment, analyzes athletes' selection of confrontation/non-confrontation strategy based on game theory and explains athletes' correct selection of confrontation/non-confrontation strategy in proper time. Before the athlete carries out the strategy, the situation on the field should be analyzed in a comprehensive and scientific way. The proper competition strategy is chosen according to such situation. In addition, they give play to their five-element ability and integrate the five elements flexibly. Finally, they adopt the optima strategy according to the competitive relation with other participants to win the competition.

\section{References}

[1] Li Shiying, Quantitative analysis of application status of competitive martial art attack and defense tactics. China Sport Science and Technology, 2000. 36(3).

[2] Zhao Gunagye, Study on methods and means for China competitive martial art training team to prepare for the 13th Asian Games. China Sport Science and Technology, 2001.37(4).

[3] Zhao Guangsheng, Gao Binghong, Guo Yuheng et al., Research on anaerobic metabolism ability feature of excellent martial art free combat athletes. China Sport Science, 2006,3:46-49.

[4] Liu Jinsong, Peng Yanming, Physiological analysis and training of free combat sport quality. Wuhan Science, 2004,12:48-50.

[5] Wu Zhaoxiang, Wu Yuhua, Generality of essential quality of martial art free combat athletes. Journal of Anhui Sports Science, 2004, 9:44-45. 